\title{
Physics and the Phenomenal World
}

\section{Jean Petitot and Barry Smith ${ }^{1}$}

Preprint version of paper published in R. Poli and P. M. Simons (eds.), Formal Ontology, Dordrecht/Boston/London: Kluwer, 1997, 233-254.

\begin{abstract}
:
The paper challenges the assumption, common amongst philosophers, that the reality described in the fundamental theories of microphysics is all the reality we have. It will be argued that this assumption is in fact incompatible with the nature of such theories. It will be shown further that the macro-world of three-dimensional bodies and of such qualitative structures as colour and sound can be treated scientifically on its own terms, which is to say not only from the perspective of psychology but also ontologically. A new sort of emergentist position will be defended, one which yields the basis of a method for describing the perceptually salient macroscopic world in mathematical terms. Broadly, it will be argued that the macroscopic world exists in virtue of certain specific sorts of boundary-patterns in the field of what is captured by the theories of microphysics.
\end{abstract}

\section{Introduction}

One of the main problems of the philosophy of science is to arrive at a plausible conception of the relations between (1) the phenomenal or commonsensical world that is apprehended in perception and described by natural language and (2) the world of standard physical theories, or of such fundamental theories of the microstructure of matter and radiation as Newtonian mechanics, the Maxwell theory of electromagnetism, special and general relativity, and quantum mechanics. The rise of mathematical physics has long been seen by many as dictating a dismissal of the phenomenal world - the world macroscopically organized in objectual forms, shapes, secondary qualities and states of affairs - from the realm of properly ontological concerns and as dictating a concomitant 'psychologization' of phenomenal structures. There is, then, a reductionist assumption common amongst philosophers to the effect that it is only microphysical reality that has a structure of its own (that the world as it is in itself is a matter of 'minute, widely-separated colourless particles' ${ }^{2}$ ). In fact, however, the discovery of atoms or quarks in no way served to eliminate molecules, macromolecules, or indeed macroscopic objects together with their macroscopic properties from the realm of physics - all are physical systems of a perfectly well-defined sort. Moreover, recent developments in cognitive science and elsewhere have given rise to a new theoretical relevance of this phenomenal or qualitative level of objective reality. We can point, for example, to the idea of a 'naive physics' such as is propagated by Patrick Hayes (1985), to the qualitative physics of Kleer and Brown (1984), as also to the earlier work on perceptual salience of J. J. Gibson (1979). ${ }^{3}$ Most scientific work on the phenomenal or

1. Our thanks go to Eero Byckling (Helsinki) for valuable suggestions.

2. Jackson 1977, p. 121.

3. See Smith 1993a; for a more detailed treatment of the history of naive physics in general see Smith 1993 and Smith and Casati 1993. 
qualitative world is however for obvious reasons psychological in orientation. ${ }^{4}$ Our thesis here, in contrast, is that it is possible to develop a coherent theoretical understanding of this world as a matter of objective structures in a sense to be more precisely determined below.

Already by looking back to the work of pre-Galilean philosophers we can gain some idea of how a theory of the common-sense world ought to go. It must, it seems, amount to one or other form of Aristotelian ontology, in the sense of an ontology recognizing enduring animate and inanimate substances manifesting an opposition between form and matter, possessing sensible and non-sensible qualities and undergoing changes of various natural and non-natural sorts. The ontology must in addition recognize species and genera (or 'natural kinds') which these entities, both substances and their accidents, instantiate, and it must recognize that the instances of these species are divided in each case into circles of more and less standard or typical instances. ${ }^{5}$

For Galileo and his successors, in contrast, substances and sensible ('secondary') qualities came to be eliminated from the realm of that which enjoys autonomous existence, along with the whole concomitant apparatus of natural kinds, prototypical instances, and so on - and it is from this perspective one of the most striking features of recent work in naive physics that a fundamentally Aristotelian apparatus is in different forms in process of being once more resurrected. ${ }^{6}$

Clearly, however, the phenomenal or commonsensical ontology - we shall for the moment treat these terms as synonymous - can be Aristotelian only in a broad sense. Thus the space of this ontology is threedimensional and global in type, as contrasted with the purely local space of Aristotle. Substances occupy volumes of this space and move continuously through it; they have closed spatial boundaries which delimit and separate them from other substances, and so on.

How, now, are we to determine the relation between commonsensical ontology and physics of the standard sort? Modern epistemology has concentrated overwhelmingly on reductionistic answers to this question. Thus at the one extreme is the physicalist trend, characteristic in particular of the thinking of some members of the Vienna circle, which strives to eliminate all that would be specific to the phenomenal realm. At the other extreme one has a variety of attempts, beginning with Mach, the early Carnap, and the later Husserl, to reconstruct physics itself on a sensory or phenomenological basis. Here, in contrast, our aim shall be to throw new light on the relation between physics and phenomenal reality in a way which takes each side of this relation seriously on its own terms. The approach that results will be compatible with physics, though not with any physicalist reductionism of the

4. See e.g. Bozzi 1958, 1959, 1989, Forguson 1989.

5. As the Gestaltists have shown, the prototypical instances in each species are more readily discriminable (salient, prägnant) than are the non-prototypical instances, and they are more readily able to give rise to correspondingly skilled responses on the parts of perceiving and acting subjects.

6. Consider, for example, the extent to which Hayes' list of relatively isolated conceptual 'clusters' or sub-theories of naive physics (1985, p. 18ff.) corresponds to the original master-list supplied by Aristotle in the Categories. 
more familiar sort. It is also more cautious than is customary in its account of the reality that is supposedly captured in physical theories.

\section{Manifestations of Matter I: Spatial Movement}

Modern physics is, crudely defined, a science of matter. It deals with a rather limited number of ways in which matter manifests itself in phenomenal reality (above all, of course, in the controlled contexts of laboratory experiments). Moreover, it deals with these manifestations not as denizens of the phenomenal world but as it were in purified form, as quantities or magnitudes: qualitative data are treated via mathematical algorithms and concepts. It seeks to use mathematical devices to explain the given manifestations by showing how they are consequences of formal laws or principles. Phenomenal reality comes thereby to be filtered entirely through structures of a formal and quantitative sort. The resultant physical models capture only a limited set of the features of phenomenal reality, and many qualitative and morphological structures of phenomenal manifestation are lost to view as such. This is not, as might be supposed, a trivial matter, a consequence of the selective attention that is characteristic of all sciences. Rather, as we shall see, the very entities with which physics deals are in certain precise ways shaped and constrained by the filtering structures with which the physicist is compelled to operate.

Classical mechanics, to take the clearest example, seeks to explain in a mathematical way, and in a single, unified framework of principles, all the diverse expressions of that manifest property of matter which is spatial movement, from the movements of pendulums and the orbits of planets to turbulence in fluid dynamics and the thermodynamic phenomena (such as heat diffusion) which are captured by statistical mechanics. Such movements are represented within the theories of mechanics either as vectors (in the case of velocities, gradients, accelerations, etc.), or as tensors (of angular momentum, of deformation in continuous media, etc.), or as differential forms (flux, divergence, curl, etc.). Vectors, tensors and differential forms are all mathematical entities which possess intrinsic geometrical meanings in the sense that they are independent of the coordinate frames we use to describe them. Here the admissible changes of coordinate frames depend in each case on the level of structure which is relevant. These changes form a group of symmetries which is characteristic of this level. For instance, for vectors the group is the linear group (or the orthogonal group when metrical properties are relevant). For inertial frames in Galilean kinematics it is the Galilean group. For differential forms it is the group of diffeomorphisms, etc.

One consequence of this, is that the descriptions of movement yielded by classical mechanics must be independent of whatever we happen to choose as co-ordinate frame among those co-ordinate frames allowed by the characteristic group. This is an a priori (which is to say pre-physical) requirement on the descriptions of the theory. Thus in Galilean kinematics the differential entities of which the theory treats must enjoy the specific mathematical property that they vary covariantly with respect to the Galilean relativity group. Or again: because no point in time is distinguishable physically from any other, it is impossible physically to determine an absolute origin of time: with this fact is associated the relativity sub-group of time-translations. Similarly, it is impossible physically to determine an absolute origin for the co-ordinates of space, or an absolute direction in space, and with these geometrical facts are associated respectively the relativity sub-groups of spatial translations and spatial 
rotations. It is impossible physically to select an absolute inertial frame; with this kinematical fact is associated the group of Lorentz transformations, and so on.

The Galilean group is a group of symmetries of space and time: it determines what is called a 'homogeneous' structure within which every point in space or time is indistinguishable from every other. In general, symmetries separate the quantities which are used in the description of a physical system into two kinds. One the one hand are quantities which are invariant under the transformations specific to the relevant physical system. (If, for example, we take a particle as our physical system, then its mass is a quantity of this sort.) On the other hand are quantities which are not invariant but are rather dependent upon the choice of coordinate system: thus for example we choose arbitrarily a zero for time, a zero for space, a geometrical co-ordinate frame, and an inertial frame. A mechanical system, now, is completely described by a certain function, called its Lagrangian, which expresses the 'action' of the system. ${ }^{7}$ One of the greatest theorems of classical mechanics, namely Noether's theorem (which can be generalized to physical theories of many other sorts), says that, if the Lagrangian is invariant through a given group of coordinate transformations, then there are certain physical quantities correlated therewith, which are conserved through every movement of the system. ${ }^{8}$ Such conserved quantities are called the first integrals of the system. Their role is fundamental in solving the Euler-Lagrange (or Hamilton) equations which are satisfied by the system and whose solutions are temporal trajectories of the system.

Noether's theorem tells us (more precisely) that to every one-parameter group of symmetries of the Lagrangian there is correlated a law of conservation of a physical quantity. A one-parameter group of symmetries is a group of symmetries of dimension one, for example the group of spatial translations in some given direction. If the Lagrangian is symmetric relative to this group, then the component of kinetic momentum in this direction is conserved. Time translations are correlated in this way with the law of conservation of energy. Spatial rotations are correlated with the law of conservation of angular momentum. From correlations of this type, when appropriately applied, we can derive deep physical predictions - to the extent that many physicists will in fact claim that the whole physical content of classical mechanics is exhausted by such laws of conservation. Einstein's celebrated law of the equivalence of mass-energy is itself a direct consequence of Noether's theorem applied to the Poincaré group, which is the relativity group of four-dimensional Minkowskian space-time, and Noether's theorem has played an ever more important role in modern physics, not only in mechanics but also in quantum field theory. ${ }^{9}$

In order to express physical laws mathematically one needs frames of reference. But these frames themselves are pre-physical conditions of description; insofar as they are a matter of conventional fixing on our

7. One can also consider the Hamiltonian of the system, which is an expression of the energy the system contains.

8. See Abraham-Marsden 1978 and Arnold 1989.

9. See on this Petitot 1992. 
part, they reflect no objective peculiarities on the side of reality. Thus although for the purposes of objective description one must for example use co-ordinate frames, such frames are in general eliminable in the sense that they lead in most cases to descriptions that are covariant under admissible changes of co-ordinates. It is an implication of our remarks above, however, that this fact has strictly physical consequences, i.e. consequences relating to what the objects are (determined by the conservation laws) with which the theory deals. It is in this context that we are to understand what Clifford has in mind when he says that 'physics is geometry' and Einstein when he says that 'objectivity is covariance'. For the phenomena of physics, both in classical and modern physics, can to a large extent be described using geometrical concepts. In the resulting models, it is found that the physically important quantities are precisely those which are invariant under transformations of the sort referred to above; such quantities are intrinsic to the physical system. When a theory is generalized, as when classical mechanics is replaced by relativity theory, the relevant group of transformations becomes larger. The resulting description is then more exhaustive, and quantities which were previously unrelated are found to be related by law.

Physics is in this sense required to absorb within itself ever more geometrical structures. For instance in classical Newtonian mechanics, force possesses a real (non-relative) physical content (because acceleration is invariant under Galilean transformations). In general relativity, on the contrary, force becomes a relative quantity, as does velocity. This is so, because the relevant relativity group is now the huge group of diffeomorphisms of space-time. The covariance principle is therefore more constraining, since invariance has to be guaranteed through a much larger family of transformations. ${ }^{10}$

\section{Physics vs. Ontology}

With relativity groups and Noether's theorem we see a shift in modern physics away from ontological concerns of the more traditional sort. For Leibniz, matter in the physical sense still had substances and a materia prima underlying it, and mechanics was only the mathematical description of one aspect of the ways in which such substances appear. In post-Newtonian (classical) mechanics, however, this Aristotelian world of substances disappears. Thus for Kant, in his Metaphysische Anfangsgründe der Naturwissenschaft, the category of substance is seen as expressing no more than the condition of the possibility of permanence of physical quantities. The concept of substance comes therefore to enjoy a largely normative role, as a way of lending system and mathematically expressible organization to the phenomena via conservation laws.

A second aspect of the post-Galilean shift away from the Aristotelian ontology is reflected in the move from qualitative to quantitative aspects of reality. An 'observable', in modern physics, must be measurable. But for something to be measurable, there has to be the possibility of conservation in certain ideal conditions.

10. In contemporary gauge theories it is the physical content of interactions which is reduced to covariance principles. Here the quantum numbers (see $\S 4$ below) are treated as spatio-temporally varying quantities. For this, the geometrical descriptions of physical systems must use not only the usual space-time geometry but more complex structures which are called fiber-bundles over space-time. The relativity group (which is called the gauge group) is now still bigger, and the covariance principle therefore still more constraining. See for instance Quigg 1983 and Petitot 1992. 
Simplifying somewhat, we can say that it is as if the necessary stability is imposed on the phenomena, and in such a way that the sort of stability we impose will determine the sort of theory we end up with.

We can now see why physics of the post-Galilean sort is not capable of serving as an ontology in the classical sense. Post-Galilean physics involves, for better or worse, an ineliminable Kantian dimension. Indeed some mathematicians and physicists, and above all Poincaré, have claimed that relativity and invariance groups are the modern form of the Kantian synthetic a priori. Modern physics yields a quantified and conceptual-formal reconstruction of reality, a unified system of mathematical regularities in the manifestations of matter, a reconstruction that is in no small part dependent upon constraints which must be satisfied if the relevant quantities are to be graspable at all. Yet still, this Kantian dimension is not of a psychological and cognitive nature: it is linked with the existence of symmetries in geometry and physics. As Leibniz already understood, symmetries say something fundamental about the nature of physical phenomena: indiscernibility is not merely an inadequacy on our part, it is a property of the physical system.

\section{Manifestations of Matter II: The Wave Function}

Matter manifests itself phenomenally not only via mechanical movement but also, for example, via the sort of behaviour captured by a wave function. Quantum physics can be seen as the physics which relates precisely to this mode of manifestation of matter, just as classical mechanics relates to movement. In addition to 'external' spacetime, quantum mechanics deals with what are called 'internal' quantum numbers. These are new physical quantities which characterize the states of elementary particles (electric charge, isospin, charm, colour, etc.). And here again there are certain constraints which prove to be of significance in determining the nature of the objects of the theory which results. For example it turns out empirically that in the nexus of strong nuclear interactions the proton and the neutron are indiscernible. The symmetry between the two is called the isospin symmetry. Applying Noether's theorem we are able to derive from this symmetry a conservation law which is the law of conservation of isospin in nuclear reactions.

Another, perhaps even more impressive, derivation of this sort turns on the fact that it is impossible by physical means to individuate an elementary particle in a group of elementary particles of the same type within a single quantum system (for example one electron in an atom with many electrons). This fact, again, seems at first not to have much physical content. The Lagrangian (or the Hamiltonian) here becomes an operator which operates on the wave function describing the quantum state of the system. This Lagrangian is invariant in respect to the symmetry that is represented by the group of permutations of the particles within the system. In some cases permuting the particles leads to no change in the function: the function is symmetric. In other cases such permutation leads to a change of sign: the function is antisymmetric. This opposition is now reflected in those physical properties of matter which are known in quantum mechanics as the correlation between spin and statistic.

Those systems which are antisymmetric are constituted by particles - called 'fermions' - which have a half-integral spin $(1 / 2,3 / 2,5 / 2$, etc.). Such particles, which are particles of matter, are subject to Pauli's exclusion principle, which states that two fermions in the same position in space-time cannot have the same quantum 
numbers. It is this principle which explains, for example, why all the electrons of an atom must have different systems of quantum numbers (electrons are fermions); and this explains in turn why one needs different orbits of electrons, why matter does not collapse, and therefore also why matter can bunch macroscopically in a smooth and stable manner and so manifest chemical properties.

Those systems, on the other hand, which are symmetric, are constituted by particles of integral spin ( 0 , 1,2 , etc.). These particles, called bosons, are particles vehiculating interactions between particles of matter. The photon, for example, is the particle vehiculating electromagnetic interaction between electrons, protons, etc. For the bosons, Pauli's exclusion principle is not valid. Thus we can have superposition of bosons in the same position in space-time, which explains such fundamental physical phenomena as lasers, superconductivity and superfluidity. Here also, therefore, we have deep, indeed quintessential properties of matter, which are in a certain sense the physical translation of certain constraints pertaining to symmetry and indiscernibility.

\section{Manifestations of Matter III: Qualitative Discontinuities}

On the one hand, then, we have objective physical determinations of different modes of manifestation of matter (movement, radiation, etc.), and on the other hand we have phenomenal (qualitative, morphological) manifestations in the sense familiar to us all pre-theoretically. Our thesis here is that phenomenal manifestation is also a mode of manifestation of matter and that there can indeed exist a sort of phenomenal physics. This phenomenal physics is of course different from standard fundamental physics: it is qualitative, macroscopic and emergent. Yet it is, nonetheless, objective.

There are well-understood ways in which physical theories can be enriched in order to capture the features specific to phenomenal reality. For physics, though in great part restricted to the quantitative, does indeed deal with just the manifestations of matter - colour, sound, temperature - from out of which the qualitative, phenomenal world is built up. Physics is not, however, interested from the theoretical point of view with those very special sorts of ways in which manifestations of matter are composed or knitted together which are relevant to the world of qualitative experience. Our task here, therefore, will be that of devising a science of salience in this sense, i.e. a science of the properly qualitative modes of manifestation of matter, with the goal of bridging the gap between quantity and quality, or between the physical and the phenomenal modes of manifestation of matter in such a way as to make the latter, too, able to serve as the object of a genuine theory.

We shall attempt to explain the qualitative structure of a phenomenon as emergent in relation to the physical behaviour of the underlying material substrate. For a property on a structure to be emergent we need three things:

1. There must be two levels of reality, a microlevel and a macrolevel, and the emergent property needs to be a property of objects on the macrolevel.

2. Objects on the macrolevel must be made up of objects on the microlevel as their parts, so that we must be able to explain causally the emergent structure exclusively by appeal to phenomena on the microlevel (causal reductionism). 
3. But on the other hand we must be able to show that there are holistic and structural or organizational features (morphological properties, properties of self-maintenance, etc.) which are distinct from those structures or organizational features which are proper to the microlevel and captured by the corresponding microlevel sciences.

Here the obvious suggestion is that the qualities manifested locally in phenomenal reality be represented as degrees of appropriate intensive magnitudes: colours via frequencies and reflectances, qualities of hot and cold via temperatures, etc. Such representations will most importantly preserve the spatial or temporal variations in the represented qualities, and it seems reasonable to suppose that it is in such variations that the relevant qualitative information will be concentrated. But only some types of physical phenomena will be able to sustain variations of the appropriate sort. Simple mechanical systems (pendulums, for example) fall out of court in this regard. On the other hand for electrical fish, charge is a qualitative phenomenon. Intuitively speaking we can say that 'qualitative' structures exist where certain fine-grained microstructures are just sufficiently smooth to admit a coarse-grained morphological organization via discontinuities (boundaries) on the macroscopic level.

Which microstructures and which associated sorts of discontinuities come into question here depends in part upon accidental features of the human perceptual system. The key theoretical idea, however, which is due to René Thom, ${ }^{11}$ turns on the opposition between 'smooth' and 'boundary' regions in the relevant spheres of variation in intensive magnitudes. Thom's idea is that the science we require should take as its main primitive qualitative discontinuity, which is to say discontinuous variations in qualities (appropriately smooth quantitative variations) as represented in the given fashion. The theory which results would then be a science of those manifestations of matter which are associated with macroscopic discontinuities of variation in intensive magnitudes in something like the way in which classical mechanics is a science of those manifestations of matter we call spatial movement.

The steps involved in building up a science of phenomenal reality along these lines can here be sketched only in broad terms:

i. We must convince ourselves that the given primitive does yield the central elements of a science of the relevant sort.

ii. We must give a mathematical expression of the idea of qualitative discontinuity.

iii. We must give an account of how we can use this idea to facilitate the move from standard physics to the science of phenomenal reality. ${ }^{12}$

We shall deal with each of these in turn.

11. See Thom 1972, 1980, 1990 and also Petitot 1985, 1992a. In his 1978 Thom distinguished two complementary aspects of an objective phenomenon: as an object of physical experience, a phenomenon 'admits as underlying symmetry group one of the groups of automorphisms of space-time'. As a manifested structure, however, a phenomenon is the result of a certain type of irreversible process (which Thom has specified mathematically) and is 'characterized by observable discontinuities'.

12. Compare the problem of moving from (laws of) physics to (laws of) psychology, as discussed e.g. in Crane 1991. 
$A d$ i: How do things appear in phenomenal reality? We begin by drawing attention to three characteristic features of the ways things, events, etc. appear in sensation and perception, features that were first clearly isolated by Husserl:

1. The things which appear to us phenomenally (which are observable), appear always from one side, present one face or aspect, and are correspondingly foreshortened or 'adumbrated'.

2. Whatever appears, appears in such a way as to manifest a foreground-background structure.

3. Whatever appears, appears in the context of a spatio-temporally extended whole. ${ }^{13}$

Take, for example, a black dog with brown spots as this appears in visual perception. Here the perceiving subject is responsible, as it were, for fixing the frame (the perspective, the point of origin) within which the contours of the dog at any given time appear. Each such frame is that portion of space that is visually accessible to the relevant subject at the relevant time. (The granularity of discontinuities which can be detected in any given case will of course depend also on the subject's powers of discrimination. This aspect however we shall here leave out of account.) First among these discontinuities, now, is the outer contour (exterior boundary) of the dog as this appears within the relevant frame (a matter of projective geometry, with the relevant observer as projection point). ${ }^{14}$ The apparent contours of the dog as a whole are a certain sort of discontinuity within the plane (frame) determined by the perceiving subject: not a discontinuity between two different qualities, but a discontinuity between a quality of the appearing thing and the qualities of the background running on behind it. Finally we have the boundaries within the (apparent plane of) the dog itself; and again, each of the apparent colours on the surface of the dog has a certain spatial extension.

It is in the context of his treatment of this feature that Husserl, in a crucial passage of the 3rd Logical Investigation ( $(8)$, explains the concept of qualitative discontinuity. Setting out from the ideas of his teacher, the psychologist Carl Stumpf, Husserl points to an opposition between what he calls 'fusion' (Verschmelzung) and 'separation' of sensible qualities. Two neighbouring qualities are fused, phenomenally, if there is no observable separation between them (as for example in a smooth transition from a darker to a lighter shade of one colour or indeed from one colour to another). Separation, in contrast, is identified precisely with discontinuous variation. A sensible phenomenon is set into relief in relation to other phenomena, now, only where a discontinuity of this sort has been created by the qualitative moments which fill its extension:

If a content is intuitively separated in relation to co-existing contents and does not flow over into these 'indistinguishably', then it can make itself count on its own and be noticed (stand forth for itself). The

13. There is, in other words, a relation of foundation in the Husserlian sense between sensible qualities and spatiotemporal extension (no colour can, as a matter of necessity, exist without spatial extension, no sound without duration, etc.). See Husserl's 3rd Logical Investigation, as also the papers collected in Smith (ed.) 1982, Fine 1993, and ch. 3 of Petitot 1992a.

14. The fact that we can only perceive one such contour at a time is a typical example of foreshortening or adumbration, as also is the fact that this apparent contour approximates rather to a one-dimensional than to a fully three-dimensional structure. This point has been emphasized by Marr (1982). See also Petitot 1990 for the geometrical theory of apparent contours. 
intuitively unseparated content, on the other hand, forms a whole with other co-existing contents, and because it is not marked off in the given manner it is not merely bound up with these contents but 'fused' therewith. ${ }^{15}$

It is separation, in other words, which accounts not only for the salience but indeed for the very existence of an item in phenomenal reality. ${ }^{16}$ Thus we have strong grounds for supposing that qualitative discontinuities can indeed serve as one central organizing principle of the phenomenal world.

$A d$ ii: In giving an appropriate mathematical expression to the notion of qualitative discontinuity we follow the topological approach outlined by Thom (1978) and developed further in Petitot (1992a). Suppose that W is the spatio-temporal extension of a given phenomenon (the dog, as this appears to a given subject at a given time). As a portion of space-time, $\mathrm{W}$ is of course a topological space with the usual topology. Suppose further that the different qualities which fill $\mathrm{W}$ are expressed by degrees of $n$ distinct intensive magnitudes $q_{1}, q_{2}, \ldots, q_{\mathrm{n}}$. The $q_{\mathrm{i}}$ are functions $q_{\mathrm{i}}(\mathrm{w})$ of points $\mathrm{w} \in \mathrm{W}$. They are the sensible qualities (colour, texture, temperature, reflectance, etc.), but considered as immanent to the objects themselves. ${ }^{17}$

A point $\mathrm{w}$ is called regular if all $q_{\mathrm{i}}(\mathrm{w})$ are continuous in a neighbourhood of $\mathrm{w}$. Let $\mathrm{R}$ be the set of regular points of W. R contains a neighbourhood of every one of its points and hence it is an open set of W. Let $\mathrm{K}$ be the complementary set of R relative to $\mathrm{W}$. $\mathrm{K}$ is the closed set of non-regular points $\mathrm{w}$, which are called the singular points of $\mathrm{W}$. Hence $\mathrm{w}$ is a singular point if there is at least one quality $q_{\mathrm{i}}$ which is discontinuous at $\mathrm{w}$. We shall call $\mathrm{K}$ the morphology of the phenomenon that fills $\mathrm{W}$. $\mathrm{K}$ is then the system of qualitative discontinuities - the pattern of boundaries - which sets this phenomenon into relief and makes it salient as a phenomenon. (Consider, for example, the morphological organization of a leaf, or of a dog, or of a wedding-photograph.)

This topological definition of the concept of morphology is as it stands purely phenomenological. It is completely neutral as to what might be the cause or the principle of production of the phenomenon or what might be the realities underlying it. In order to accord physical content to the definition, we must now find some way to conceive a morphology $(\mathrm{W}, \mathrm{K})$ as a manifestation of physical properties internal to whatever underlies or causes the phenomenon in question.

Our thesis, now, is that the perceptually salient macrolevel objects are constituted by certain sorts of boundary-patterns to which the physical substrate gives rise. Note that such patterns, together with the phenomenal

15. See Husserl 1975/84 (vol. II, A239, B244). The ideas in this work reveal a topological sophistication which is unfortunately lost in the English translation; for this and other reasons Husserl's ideas in this connection have been until recently ignored by his successors.

16. Cf. Meinong's discussion of the 'prerogative of difference' in $\S 22$ of his 1906.

17. Of course certain simplifications are involved here. Thus colour might be accounted for in a detailed account in terms of three distinct qualities: hue, saturation and brightness. Similarly there is no single property of reflectance; the latter is rather a macroscopic approximation of a more fine-grained system on the quantum level of a range of properties having to do with the emission-absorption spectra of the atoms constituting the substrate. 
items which they circumscribe, exist independently of human perceiving subjects. They are objective; but they are of no intrinsic physical interest because they play no role in properly physical explanations at the microlevel. Moreover, they would be of no interest at all were it not for the existence of subjects whose perceptual organs are tuned in correspondence to them.

Ad iii: We can understand such boundary-patterns more precisely as follows. In many standard physical descriptions, the instantaneous states of a system with $n$ degrees of freedom are represented by points $\mathrm{x}$ of what is called a 'phase space' $\mathrm{M}$, which is a differentiable manifold of dimension $n$. For example for a system of $\mathrm{N}$ particles in 3-dimensional space, the phase space is the $6 \mathrm{~N}$-dimensional space of the positions and velocities of the particles. For a chemical system of $\mathrm{N}$ chemical substances in interaction, the phase space is the $\mathrm{N}$-dimensional space of concentrations of the substances. For a magnetic system of $\mathrm{N}$ atoms $\mathrm{a}_{\mathrm{i}}$ with spins $\sigma_{\mathrm{i}}$ (or, in an analogous manner, for a neuronal network of $\mathrm{N}$ neurons $\mathrm{a}_{\mathrm{i}}$ with states of activation $\sigma_{\mathrm{i}}$ ) the phase space is the $\mathrm{N}$-dimensional space of the families $\sigma=\left(\sigma_{\mathrm{i}}\right)_{\mathrm{i}=1, \ldots, \mathrm{N}}$, etc. ${ }^{18}$

The dynamic of the system, now, is described by means of a system of ordinary differential equations, or in other words a dynamical system on M. ${ }^{19}$ Suppose that $\mathrm{X}$ is such a dynamical system on the phase space M. From each instantaneous state $\mathrm{x}$ the system follows a certain trajectory in $\mathrm{M}$. The instantaneous states taken individually are transient: they are too fleeting to be observable. The effectively observable states of a system correspond rather to the asymptotic and stable behaviours of trajectories where the energy is minimized. Such effectively observable states and behaviours, those states and ways of behaving into which the system falls under normal conditions, are called the attractors of the system. The simplest case of an attractor is a stable equilibrium point: think for example of the rest-position of a dissipative pendulum. Starting from a position away from this point, the pendulum oscillates but little by little approaches the stable position. Its trajectory is asymptotically attracted by this fixed point. Or consider an oscillating electric circuit: from any initial state the system after some time reaches the stable oscillatory state and so its trajectory is attracted by this state.

Return, now, to our phenomenon having substrate S, spatio-temporal extension $\mathrm{W}$, and morphology $\mathrm{K}$. Here the phase space $\mathrm{M}$ is the space of local physical states of the substrate S. Suppose that the physical behaviour of the substrate at each point $\mathrm{w}$ is physically describable by some dynamical system $\mathrm{X}_{\mathrm{w}}$ on $\mathrm{M}$. Then we can move from the local to a global point of view, and assert that the mapping

$$
\sigma: \mathrm{w} \longrightarrow \mathrm{X}_{\mathrm{w}} \text {, }
$$

from the extension $\mathrm{W}$ to the functional space of the possible dynamical systems on $\mathrm{M}$ expresses the internal properties of the substrate of the phenomenon taken as a whole. $\mathrm{M}$ is referred to as the internal space of the system, $\mathrm{W}$ as the external space. $\mathrm{X}_{\mathrm{w}}$ is the internal dynamic of the system at $\mathrm{w}$, and the attractors of $\mathrm{X}_{\mathrm{w}}$ are the

18. For an elaboration of morphological ideas in the treatment of connectionism see Petitot 1991.

19. See again Abraham and Marsden 1978; Arnold 1989. 
internal states of the system at w. We now have enough machinery to explain physically how it is possible that in the external space $\mathrm{W}$ a morphology $\mathrm{K}$ can emerge.

Choose $\mathrm{w} \in \mathrm{W}$. Let $\mathrm{X}_{\mathrm{w}}$ be the internal dynamic at $\mathrm{w}$. In the general (normal) case, the internal state of the substrate $\mathrm{S}$ at $\mathrm{w}$ is physically described by an attractor $\mathrm{A}_{\mathrm{w}}$ of $\mathrm{X}_{\mathrm{w}}$. The phenomenal qualities $q_{\mathrm{i}}(\mathrm{w})$ are intensive quantities associated with $\mathrm{A}_{\mathrm{w}}$. To explain the qualitative discontinuities of the $q_{\mathrm{i}}(\mathrm{w})$, we now let $\mathrm{w}_{\mathrm{o}} \in \mathrm{K}$ be some singular point of $\mathrm{W}$. We consider a path $P$ crossing $\mathrm{K}_{\text {at }} \mathrm{w}_{\mathrm{o}}$. The idea is that in moving through points $\mathrm{w} \in P$ the attractor $\mathrm{A}_{\mathrm{w}}$ becomes unstable when we cross $\mathrm{w}_{\mathrm{o}}$. That is to say, it is replaced suddenly by another attractor $\mathrm{B}_{\mathrm{w}}$. In the theory of dynamical systems such a phenomenon is called a bifurcation of attractors. When a system is subject to such a bifurcation, it is subject also to a sudden transition of its internal state and so manifests a qualitative discontinuity. Similarly we can explain the exterior boundaries of things by saying that when we cross such a boundary, the internal state $\mathrm{A}_{\mathrm{w}}$ disappears entirely. Such boundaries, too, are phenomenally salient.

In macroscopical physics, there are many examples of such phenomena of transition of the internal states of a system. They are known as critical phenomena..$^{20}$ A typical example is that of phase transitions in thermodynamics, where a system undergoes a sudden change of phase (for example from solid to liquid or from liquid to gas, from a magnetic to a non-magnetic phase, from normal conductivity to superconductivity, and so on). Such changes occur when a parameter such as temperature crosses a critical value. The external space $\mathrm{W}$ is the space of (observable) control parameters such as temperature or pressure. The internal space $\mathrm{M}$ is the space of molecular states of the system, the internal dynamic $\mathrm{X}_{\mathrm{w}}$ is the molecular dynamic and the attractors are the molecular states underlying the phases. Phenomenologically, the internal space $\mathrm{M}$ and the internal dynamic $\mathrm{X}_{\mathrm{w}}$ are unobservable (their description is the task of standard physics proper). What we experience as salient (and what we possess words to describe) are the qualitative discontinuities which are the phase transitions, together with the smooth regions (for example phonemes) these discontinuities serve to mark out within the relevant phenomenal space.

There are many other critical phenomena: for example shock waves in acoustics, transition to turbulence in hydrodynamics, buckling in elasticity theory, etc. All such phenomena are salient in our perceptual experience. They are the physical support of the qualitative morphological organization of the phenomenal world.

\section{A Theory of the Commonsense World}

In this manner we can begin to heal the rift pointed out in our first section between physics and the phenomenal world. We have now at our disposal a theory which is founded in the physics of the substrates and which describes how morphologies or macrolevel boundary-patterns can emerge therefrom. This treatment of morphologies in the phenomenal world employs not the microscopic physics of fermions, bosons, etc., but the resources of a macroscopic physics only. In fact we do not take account of the relation between $\mathrm{X}_{\mathrm{w}}$ and the fine-grained physics

20. See for example the material in Domb and Green (eds.), 1972-1985 as well as Petitot 1992a (ch. 5). 
at $\mathrm{w}^{21}$ This relation is not our concern: it is dealt with by standard physical theories. We simply assume that we can describe macroscopically the relevant internal physical properties via a dynamical system, and this can indeed be shown already to be possible in very many cases.

Of course it might be argued that our 'morphological' science of the qualitative world is at the same distance from an ontology in the strict sense as are classical mechanics, quantum mechanics, etc. We are after all dealing here, too, not with objects (qualities, etc.) in the world, but with products of mathematical reconstruction. ${ }^{22}$ The reconstruction here presented allows however a direct mimicking of those central features of the Aristotelian commonsensical ontology that were so fatefully abandoned by Galileo and his successors. Thus not only can it claim to offer a sort of qualitative physics; it can also furnish a theory of substance, of change or process, and of typicality, species and categorization, as also of other pervasive features of the commonsense world. It is this which justifies our use of the terminology of a phenomenal or qualitative 'ontology'.

As concerns the theory of substance and change, here the central problem is that of understanding the relation between the perceived object itself (in our example, the spotted dog) and the family of its apparent contours. There are two abstractly distinguishable systems of continuous development in the latter, one bound up with movements of the object, the other with movements of the perceiving subject. The theory of substance is in the first place a theory of the mathematical properties of the first of these two sorts of continuous development. The geometrical problem to be solved is this: how can the object be unambiguously retrieved from the system of qualitative discontinuities which are given by its apparent contours. ${ }^{23}$

As concerns the problem of categorization and typicality, here we must distinguish two cases. If the entities to be categorized depend on a finite number of characteristic cues (as is the case in relation to colours and phonemes), then a $(\mathrm{W}, \mathrm{K})$ model, where $\mathrm{W}$ is the space of the cues, is a model of categorization. A category $\mathrm{C}$ defined by $\mathrm{K}$ is a connected component of $\mathrm{W}-\mathrm{K}$. In each category there is defined statistically a certain centre, the prototype $\mathrm{T}_{\mathrm{C}}$ of the category. As Petitot (1989a) shows, the strategy here outlined can be applied to the elaboration of very detailed models of categorial perception in phonetics (of the ways in which phonetic perception categorizes the continuous audio-acoustic flux by chopping it up into phonemes).

Take, however, the more complicated case of visual forms. Let us suppose that the latter constitute a space F. We can then distinguish within $\mathbf{F}$ the structurally stable forms as those whose qualitative type is invariant relatively to small deformations. These constitute an open set $\mathbf{R}$ of $\mathbf{F}$. Let $K_{\mathbf{F}}$ be the complementary set of $\mathbf{R}$ relative to $\mathbf{F} . \mathrm{K}_{\mathbf{F}}$ then effects a division of $\mathbf{F}$ into stable types of forms, i.e. categories or species, which are delimited from each other by boundaries made up of unstable forms.

21. Consider, for example, the case of a chemical reaction. Here we take for $\mathrm{X}_{\mathrm{w}}$ the kinetic chemical equations of the chemical constituents and not the quantum physics of the substrate.

22. For a sketch of some alternatives to this Kantian view of the qualitative morphological ontology - a view criticized in Morton 1990 - see Smith 1993.

23. See Petitot 1990. 


\section{Qualitative Ontology and the Science of Cognition}

Could something like the phenomenal or commonsensical ontology outlined above constitute a scientific theory in the full sense? Here the most important criticism would be that the theory in question is not predictive in the usual (causal) sense. It can be pointed out, however, that the approach does lead to prediction, though only in the sense that it leads to the possibility of our explicating mathematical constraints for the empirical morphologies. This is prediction of the same sort as, for example, predictions to the effect that if you have a crystal, or the envelope of a virus, or a snowflake, or a honeycomb, or an ornamentation of the Alhambra of Granada, then the symmetry of the structure is necessarily one of the abstract symmetries which are allowed by pure geometry. There exist theorems which make the same type of structural predictions for the possible morphologies K. Even if they are a variety of structural prediction or structural explanation, and not of causal prediction or explanation, these predictions can be interpreted as abstract mathematical constraints upon the universe of morphological phenomena. $^{24}$

Our thesis, therefore, is that a truly scientific theory of the phenomenal world can be rooted in the qualitative macro-physics of the material substrates. But in order to have a plausible theory of the phenomenal world we need in addition a psychological-cognitive theory of perception and an account of the link between this theory and the theory of the substrates. How is the subject involved in the perceptual explication and cognitive interpretation of the qualitative structures of the phenomenal world? As far as qualities such as colour are concerned, we already dispose of considerable work on these problems, and we know something about the chain of steps which lead from physics to the mind. We have first of all, at the microlevel, the absorption-emission spectra of the atoms making up the substrate. At the macrolevel we have the reflectance of the object, which gives rise in its turn to transmission of light of certain wavelengths. At the level of the retina, the light excites the photoreceptors and the information (pattern of wavelengths) it bears is processed by these transducers - which is to say it is transformed by the photoreceptors from photochemical into neuronal information (frequencies of neuron-firings codifying the wavelengths). This gets processed further on its way to the visual cortex, where there at last occurs the registering of the sensible quality of colour.

From our morphological point of view, now, the fundamental link between object and mind is seen as being furnished precisely by the concept of qualitative discontinuity. For this concept can be applied equally to qualities as manifested physically and as apprehended in patterns of sensation in the mind (and it is precisely for this reason that they are phenomenally salient). Wave optics explains (in a non-trivial manner) how the very special type of information provided by qualitative discontinuities can be encoded in the light (that is to say how singularities can be propagated by the light). ${ }^{25}$ Theories of visual perception for example of the sort that is

24. Perhaps the best known theorem of this sort is the Whitney-Thom-Arnold theorem classifying elementary catastrophes. But there are also other theorems concerning universal properties of critical phenomena and bifurcation scenarios, for example in relation to the routes towards chaos or turbulence via an infinite number of successive bifurcations, as in Feigenbaum's scenario. See Petitot 1992a.

25. See ch. 5 of Petitot 1992a. 
propounded by David Marr seek to clarify the perceptive endowment which allows the human cognitive system to detect and to process this information. There is strong evidence for the hypothesis that the retina makes a wavelet analysis of the signal (that is to say, a local and multi-scale Fourier analysis) and picks up the qualitative discontinuities therefrom (Marr's theory of the so-called 2-D primal sketch). Some of these 2-dimensional qualitative discontinuities are then interpreted as apparent contours of 3-dimensional objects. ${ }^{26}$

The interest of the work of Marr and his successors ${ }^{27}$ is that it reconciles two apparently antagonistic approaches: the information-processing approach and an ecological point of view in the style of Gibson. In the classical cognitivist paradigm (as exemplified by Fodor, Pylyshyn, et al.), information processing is essentially reduced to the operations of calculation on symbolic mental representations. ${ }^{28}$ These operations are essentially syntactic: the cognitivists focus exclusively on algorithms and neuronal implementations thereof, and thus, familiarly, they leave no room for the attempt to do justice to the link between the cognitive system and the qualitative features of the world outside. If, however, one wants to introduce objective structures of the environment into the account of perception and cognition (as the ecologists do), then one is committed to making such structures compatible with the information-processing devices utilized by human perceivers. This is what Marr comes close to doing, and this is why his work is a step along the road to the overcoming of methodological solipsism. Marr shows how what Gibson considered as the 'extraction' (pick-up) of invariants from the environment might be understood in information-theoretic terms as a form of computation.

A truly adequate theory, however, must not only focus on algorithms and neuronal implementations. It must in addition, as Marr himself saw, find a means of comprehending these algorithms in relation to the objective (external) type of information which they process. The algorithms must in this way be determined by objective properties of the environment. The mathematical theory of qualitative discontinuities, now, seems to offer a useful starting point for understanding the sort of determination that is here at issue: for before imagining formal algorithms for the processing of apparent contours, etc., one must know what mathematical type of information such structures consist of. And as we have argued, this information is essentially constituted by singularities: they are drawn from the family of perceptually salient boundary-patterns.

\section{References}

Abraham, R. and Marsden, J. E. 1978 Foundations of Mechanics, Reading, Mass: Benjamin/Cummings.

Arnold, V. I. 1989 Mathematical Methods of Classical Mechanics, New York: Springer.

Arnold, V. I., Gusein-Zade, S. M. and Varchenko, A. N. 1985 Singularities of Differentiable Maps, Boston: Birkhäuser.

26. Cf. Marr's theory of the so-called 2-and-a-half-D sketch, and also Petitot 1990 and ch. 3 of Petitot 1992a.

27. See e.g. Poggio 1984, Koenderink and Doorn 1986.

28. See Fodor 1980 and Fodor and Pylyshyn 1981. 
Bozzi, P. 1958 “Analisi fenomenologica del moto pendolare armonico”, Rivista di Psicologia, 52, 281-302.

Bozzi, P. 1959 “Le condizioni del movimento 'naturale' lungo i piani inclinati”, Rivista di Psicologia, 53, $337-$ 352.

-- 1989 “Sulla preistoria della fisica ingenua", Sistemi intelligenti, 1, 61-74.

Crane, T. 1991 "All God has to do", Analysis, 51, 235-244.

Domb, C. and M. S. Green (eds.) 1972-1985 Phase Transitions and Critical Phenomena, New York: Academic Press.

Fine, K. 1993 "The Theory of Part and Whole", in B. Smith and D. W. Smith, eds., Husserl, Cambridge: Cambridge University Press, forthcoming.

Fodor, J. 1980 "Methodological Solipsism Considered as a Research Strategy in Cognitive Psychology", Behavioral and Brain Sciences, 3, 63-73.

Fodor, J. and Pylyshyn, Z. 1981 "How Direct is Visual Perception? Some Reflections on Gibson's 'Ecological Approach", Cognition, 9, 139-196.

Forguson, L. 1989 Common Sense, London and New York: Routledge.

Gibson, J. J. 1979 The Ecological Approach to Visual Perception, Boston: Houghton-Mifflin.

Hayes, Patrick J. 1985 “The Second Naive Physics Manifesto”, in J. R. Hobbs und R. C. Moore (eds.), Formal Theories of the Commonsense World, Ablex, 1-36.

Husserl, E. 1975/84 Logische Untersuchungen (Husserliana, vols. XVIII and XIX), Dordrecht: Nijhoff.

Jackson, F. 1977 Perception, Cambridge: Cambridge University Press.

Kleer J. D. and Brown, J. S. 1984 “A Qualitative Physics Based on Confluences”, Artificial Intelligence, 24, 7-84.

Koenderink, J. J. and Doorn, A. J. van 1986 "Dynamic Shape”, Biological Cybernetics, 53, 383-396.

Marr, D. 1982 Vision, San Francisco: Freeman.

Meinong, A. von 1906 Über die Erfahrungsgrundlagen unseres Wissens, Berlin: J. Springer (repr. in Meinong, Gesamtausgabe, vol. V, Graz: Akademische Druck- und Verlagsanstalt, 1978).

Morton, A. 1990 “Can't Kant: Smith on Folk Physics”, in J. E. Tiles, G. T. McKee and C. G. Dean, eds., Evolving Knowledge in Natural Science and Artificial Intelligence, London: Pitman Publishing, 251-261.

Nassau, K. 1983 The Physics and Chemistry of Color, New York: John Wiley and Sons.

Petitot, J. 1985 Morphogenèse du Sens, vol. 1, Paris: Presses Universitaires de France.

-- 1989 "Morphodynamics and the Categorial Perception of Phonological Units", Theoretical Linguistics, 15, $25-71$.

-- 1989a “Hypothése localiste, Modèles morphodynamiques et Théories cognitives", Semiotica, 77, 65-119. 
-- 1990 “Le Physique, le Morphologique, le Symbolique. Remarques sur la Vision”, Revue de Synthése, 4, 139183.

-- 1991 "Why Connectionism is Such a Good Thing. A Criticism of Fodor and Pylyshyn's Criticism of Smolensky", Philosophica, 47, 49-79.

-- 1992 "Actuality of Transcendental Aesthetics for Modern Physics", in L. Boi, et al., eds., 1830-1930. A Century of Geometry (Lecture Notes in Physics 402), Berlin: Springer, 273-304.

-- 1992a Physique du Sens, Paris: Editions du CNRS.

Poggio, T. 1984 "Vision by Man and Machine”, Scientific American, 250, 68-78.

Petitot, J. and Smith, B. 1990 "New Foundations for Qualitative Physics", in J. E. Tiles, et al., eds., Evolving Knowledge in Natural Science and Artificial Intelligence, London: Pitman, 231-249.

Putnam, H. 1987 The Many Faces of Realism, La Salle: Open Court.

Quigg, C. 1983 Gauge Theories of the Strong, Weak and Electromagnetic Interactions, Menlo Park: Benjamin/Cummings.

Smith, B. 1993 “The Structures of the Commonsense World”, in A. Pagnini and S. Poggi, (eds.), Gestalt Theory. Its Origins, Foundations and Influence, Florence: Olschky.

Smith, B. 1993a "Common Sense", in B. Smith and D. W. Smith, eds., Husserl, Cambridge: Cambridge University Press, forthcoming.

Smith, B. (ed.) 1982 Parts and Moments. Studies in Logic and Formal Ontology, Munich: Philosophia.

Smith, B. and Casati, R. 1993 "Naive Physics: An Essay in Ontology", Philosophical Psychology, forthcoming.

Thom, R. 1972 Stabilité structurelle et Morphogenèse, New York: Benjamin, Paris: Ediscience.

-- 1978 “Formalisme et Scientificité”, Les Etudes philosophiques, 2, 171-78.

-- 1980 Modèles mathématiques de la Morphogenèse, Paris: Christian Bourgois.

-- 1988 Esquisse d’une Sémiophysique. Physique aristotélicienne et Théorie des Catastrophes, Paris: Intereditions.

-- 1990 Apologie du Logos, Paris: Hachette. 